\title{
Méthode de dosage du technétium 99 dans les urines par scintillation liquide
}

\author{
H. TYMEN*, N. ROBERT**, O. GIRARD DE VASSON**, \\ R. FOTTORINO**
}

(Manuscrit reçu le 15 avril 1997, révisé le 15 juillet 1997, accepté le 30 janvier 1998)

RÉSUMÉ Le technétium 99 est un produit de fission à vie longue, dont la production au cours de la combustion de l'uranium enrichi n'est pas à négliger. Des études concernant la gestion de ce déchet ont été mises en place au CEA. Celles-ci vont entraîner une exposition potentielle du personnel de recherche. Il était donc nécessaire de disposer d'une surveillance radiotoxicologique adaptée. La méthode proposée permet une détection précoce de ${ }^{99} \mathrm{Tc}$ dans les urines par scintillation liquide, en sachant que $10 \%$ de la contamination par inhalation se retrouve dans les urines dès les $\mathbf{4 8}$ premières heures après l'incorporation. Cette méthode est rapide (1 heure) et relativement sensible (limite de détection à $0,06 \mathrm{~Bq} \mathrm{~m}^{-1}$, ce qui correspond à une incorporation par inhalation égale à $8,4 \times 10^{-5}$ LAI soit $1,7 \times 10^{3} \mathrm{~Bq}$ de ${ }^{99} \mathrm{Tc}$ ).

ABSTRACT Method of Technecium 99 determination in urine through liquid scintillation. Technetium 99 is a long-life fission product; when enriched uranium is burnt, its amount is rather small but cannot be overlooked. Studies about reprocessing of this waste are being carried out by the CEA. They will lead to possible exposure to ${ }^{99} \mathrm{Tc}$ of research workers. So it seems necessary to develop a close radiotoxicological watch suited to the situation. The proposed method enables an early detection of ${ }^{99} \mathrm{Tc}$ in urine through liquid scintillation, owing to the fact that $10 \%$ of the contamination by inhalation is found in the 48 first hours urine after intake. This method is quick $(60 \mathrm{~min})$ and rather sensitive (detection is possible from $0.06 \mathrm{~Bq} \mathrm{ml}^{-1}$, that corresponds to an incorporation of $8.4 \times 10^{-5} \mathrm{LAI}$ or $1.7 \times 10^{3} \mathrm{~Bq}$ of ${ }^{99} \mathrm{Tc}$ ).

\section{Introduction}

À la suite du rapport Bataille (1991), le CEA s'est donné 15 ans pour mettre en œuvre des études concernant la gestion des déchets à vie longue. Ces études dans le cadre des Programmes CAPRA (calcination du plutonium dans les réacteurs à neutrons rapides) et SPIN (séparation et incinération des éléments

\footnotetext{
* Service de Protection Radiologique des Armées, 1 bis, rue du Lt. Raoult Batany, 92140 Clamart, France. ** Laboratoire d'Analyses de biologie Médicale, Centre d'Études de Cadarache, 13108 Saint-Paul-LezDurance, France.
} 
à vie longue) vont exposer le personnel de recherche à des produits de fission à vie longue $\left({ }^{99} \mathrm{Tc},{ }^{129} \mathrm{I},{ }^{135} \mathrm{Cs}\right)$. Une surveillance radiotoxicologique adaptée à ces radioéléments s'avère nécessaire.

La démarche de cet article est de présenter plus particulièrement une technique de dosage du technétium 99 , adaptée à la routine d'un laboratoire de radiotoxicologie dans lequel le technétium n'est pas un paramètre couramment mesuré.

\section{Le technétium 99}

Le technétium 99 n'est pas à confondre avec son isotope métastable, qui est un émetteur $\gamma$ et de très courte période radioactive très utilisé en médecine nucléaire. A contrario, le technétium 99 non métastable est peu connu. C'est pourtant un produit de fission à vie longue à ne pas négliger : en France, le combustible à base d'uranium produit chaque année au cours de sa combustion $900 \mathrm{~kg}$ de ${ }^{99} \mathrm{Tc}$.

Le tableau I indique les principales propriétés du technétium 99 (GMELIN, 1982).

\section{TABLEAU I}

Principales propriétés du technétium 99.

Main properties of technecium 99.

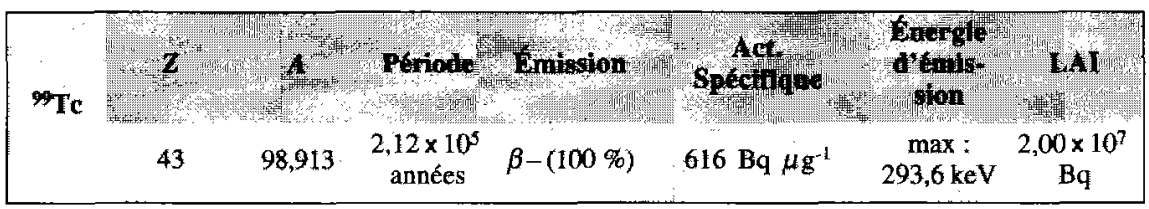

La surveillance radiotoxicologique de ${ }^{99} \mathrm{Tc}$ est moins aisée que celle de son isotope métastable. En effet, le technétium 99 n'émet que des rayonnements $\beta$ de faible énergie $(0,3 \mathrm{MeV})$, ce qui rend toute mesure directe impossible. La méthode par scintillation liquide est, pour l'instant, la technique la plus adaptée à une surveillance radiotoxicologique de routine.

\section{Matériel}

Notre méthode par scintillation liquide ne nécessite aucune préparation particulière :

- les urines sont introduites dans des flacons bouchonnés à usage unique pour scintillation liquide (flacons de $20 \mathrm{ml}$ en polyéthylène commercialisés par Packard), 
- le liquide scintillant ajouté aux urines est de l'Instagel ${ }^{\circledR}$ commercialisé par Packard (liquide de scintillation prêt-à-l'emploi à base de xylène),

- les solutions étalons ont été préparées à partir d'une source de ${ }^{99} \mathrm{Tc}$ du LMRI (concentration de $811 \mathrm{~Bq} \mathrm{ml}^{-1}$ ),

- les dilutions ont été effectuées à partir d'eau filtrée désionisée par le système Milli $Q^{\circledR}$ de chez Millipore.

Le compteur à scintillation liquide est un compteur Tri-Carb modèle 1500 de marque Packard.

\section{Méthode}

\subsection{Mise au point technique}

La mise au point de la méthode consiste à :

- choisir un rapport optimum de prise d'essai (scintillant et échantillon),

- mettre en évidence l'influence du quenching sur le rendement.

Dans ce cadre, les conditions opératoires sont identiques pour les différentes étapes :

- activité introduite dans chaque flacon : $0,1 \mathrm{ml} \mathrm{de}{ }^{99} \mathrm{Tc}$, soit une activité théorique de $81,1 \mathrm{~Bq}$ par échantillon, affectée d'une incertitude globale de $3 \%$ (compte tenu des incertitudes liées aux fluctuations statistiques de comptage, au titre de l'étalon, au volume de prise d'essai...),

- temps de comptage : $10 \mathrm{~min}$,

- sélection des canaux : plage s'étalant de 0 à $300 \mathrm{keV}$.

Le phénomène d'affaiblissement lumineux ou quenching, dû à certaines molécules chimiques absorbantes, est traduit sur le Tri Carb 1500 sous forme d'une valeur appelée «tSIE». Selon la méthode de standardisation externe, l'appareil présente une source externe $\gamma$ de ${ }^{133} \mathrm{Ba}$ devant l'échantillon à mesurer : les rayonnements gamma provoquent des électrons Compton qui ont, sur le scintillant, un effet identique aux rayonnements $\beta^{-}$. Si l'échantillon renferme un composé quenchant, le spectre dû aux électrons Compton est affaibli (Simonet et Oria, 1980). La différence entre le spectre quenché et non quenché permet au tri Carb de calculer le rapport de standardisation externe ou tSIE. Plus la valeur du tSIE est faible, plus l'échantillon est quenché, moindre sera le rendement. 
Influence du volume de scintillant.

Afin de déterminer le volume optimum de scintillant, des prises d'essai contenant $0,1 \mathrm{ml}$ de source étalon et des quantités variables d'Instagel (5 à $20 \mathrm{ml}$ ) sont soumises à un comptage de $10 \mathrm{~min}$.

Les résultats des mesures ont permis d'établir la courbe de rendement en fonction du volume de scintillant. Cette courbe, présentée en figure 1, fait apparaître que le volume de scintillant qui correspond au rendement et au tSIE les plus élevés, est de $15 \mathrm{ml}$.

Au cours des expériences ultérieures, chaque prise d'essai comportera (dorénavant) $15 \mathrm{ml}$ de scintillant.

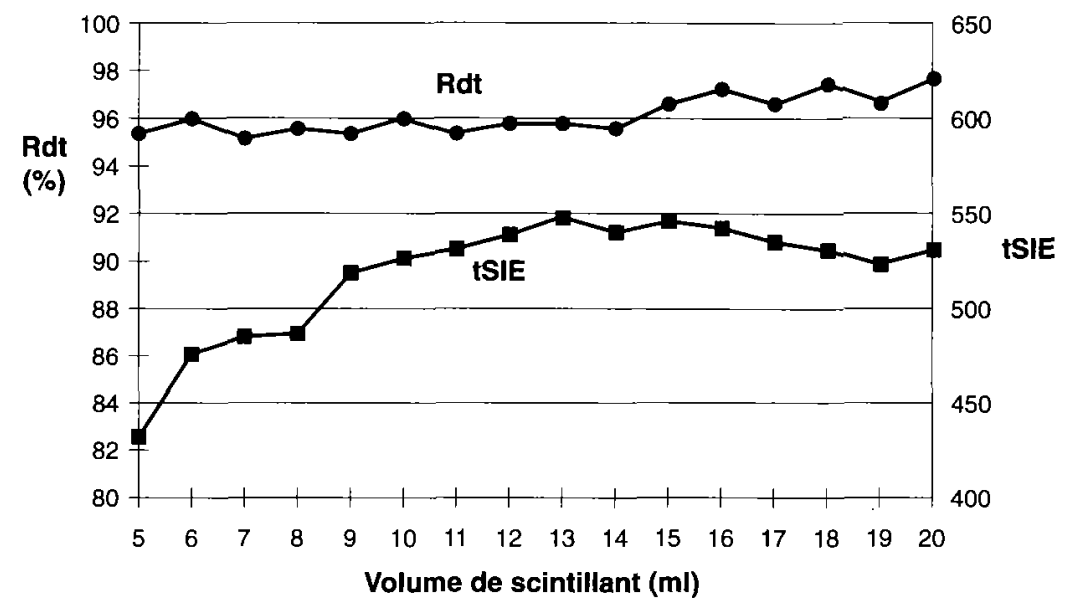

Fig. 1. - Rendement et tSIE en fonction du volume de scintillant.

Outpout and tSIE in proportion to the volume of scintillating liquid.

Influence du volume de phase aqueuse.

De nature organique, l'Instagel ${ }^{\circledR}$ est instable en présence de phase aqueuse en excès (urine par ex.). Afin de déterminer le volume optimum de phase aqueuse, des échantillons contenant $0,1 \mathrm{ml}$ de source étalon, $15 \mathrm{ml}$ d'Instagel et des quantités variables de phase aqueuse $(0,5$ à $5 \mathrm{ml})$ sont soumises à un comptage de $10 \mathrm{~min}$ (la phase aqueuse est de l'eau distillée et non de l'urine).

Les résultats des mesures ont permis d'établir la courbe de rendement en fonction du volume de phase aqueuse. Cette courbe, présentée en figure 2 , fait apparaître qu'un excès de phase aqueuse affaiblit le rendement et le 
tSIE : les faibles prises d'essai sont donc plus favorables. Nous avons opté arbitrairement pour $1 \mathrm{ml}$ de phase aqueuse par soucis d'homogénéité dans les prélèvements destinés à la mesure par scintillation liquide au sein du laboratoire.

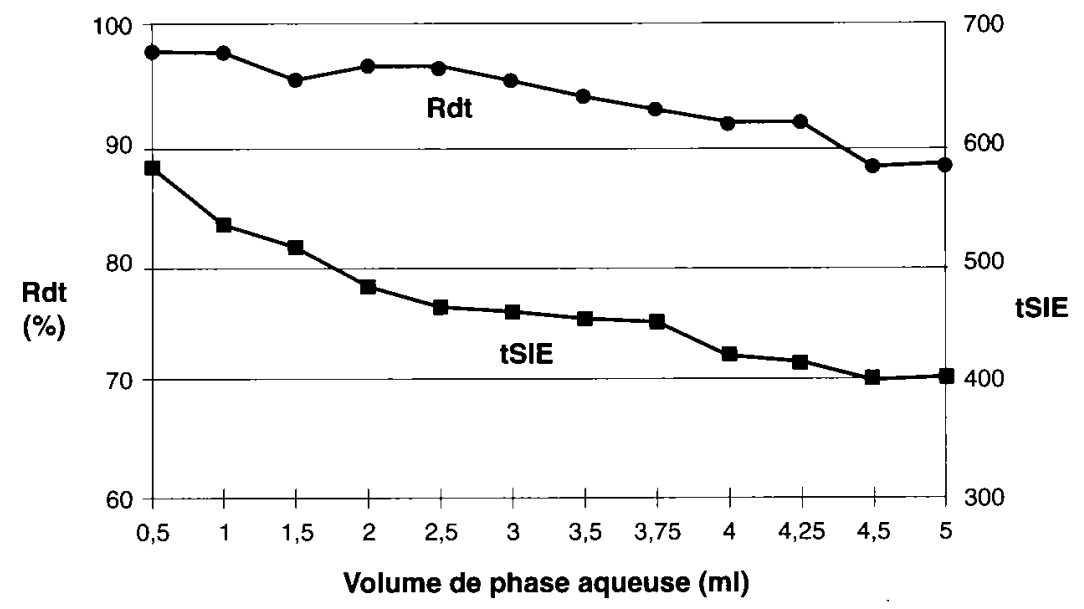

Fig. 2. - Rendement et tSIE en fonction du volume de phase aqueuse.

Outpout and $t$ SIE in proportion to the volume of aqueous phase.

Influence du quenching chimique sur le rendement.

Le phénomène d'affaiblissement lumineux n'est pas à négliger dans le cas des urines. Il peut diminuer de façon notable le rendement. La relation entre le rendement et les valeurs de tSIE est obtenue à partir d'échantillons standards quenchés : 36 flacons contenant au préalable la source étalon et $1 \mathrm{ml}$ de phase aqueuse (eau distillée) ont reçu des valeurs croissantes de tétrachlorure de carbone ( 0 à 34 gouttes de $\mathrm{CCl}_{4}$ ). Le scintillant est ajouté en dernier de telle façon à ajuster à un même volume final les 36 échantillons (soit 16,1 ml). La courbe de correction de quenching présentée en figure 3 montre que la mesure est peu sensible au quenching. Ceci s'observe dans le cas des émetteurs $\beta$ à énergie d'émission relativement importante $\left({ }^{36} \mathrm{Cl},{ }^{90} \mathrm{Sr},{ }^{137} \mathrm{Cs}\right)$. Ainsi, dans le cas du technétium, on peut s'affranchir de la courbe de correction de quenching. 


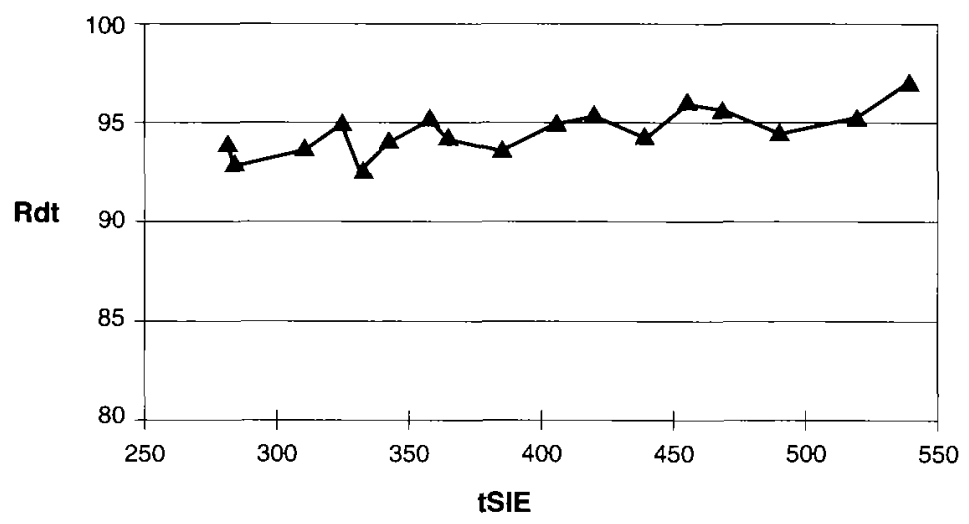

Fig. 3. - Rendement en fonction du tSIE.

Outpout in proportion to tSIE.

\subsection{Conditions opératoires retenues}

Le mode opératoire d'une analyse de ${ }^{99} \mathrm{Tc}$ dans les urines consiste donc à introduire directement dans le compteur à scintillation des échantillons composés d'1 $\mathrm{ml}$ d'urine et de $15 \mathrm{ml}$ d'Instagel ${ }^{\circledR}$. L'expression du résultat ne nécessite pas en principe l'établissement d'une courbe de correction de quenching. On peut supposer l'influence du quenching dû à la couleur relativement limitée car l'énergie d'émission de ${ }^{99} \mathrm{Tc}$ n'est pas très basse. Une précaution à signaler est de vérifier le $\mathrm{pH}$ de l'urine à analyser et de l'abaisser à 5-6 si besoin est.

\section{Résultats}

\subsection{Essais sur urines}

Détermination d'un bruit de fond moyen.

Sur 10 urines brutes, le bruit de fond moyen pour une prise d'essai de $1 \mathrm{ml}$, a été évalué à 0,40 coups par seconde.

Détermination d'un rendement moyen.

Sur 10 urines surchargées avec $0,1 \mathrm{ml}$ de ${ }^{99} \mathrm{Tc}$ à $811 \mathrm{~Bq} \mathrm{ml}{ }^{-1}$, et pour une prise d'essai de $1 \mathrm{ml}$, le rendement moyen a été évalué à $95 \pm 2 \%$. 
Valeurs du tSIE dans les échantillons d'urines.

Nous avons vu auparavant l'influence limitée du quenching chimique sur le rendement. Néanmoins, travaillant sur des urines, il nous faut vérifier l'influence du quenching dû à la couleur : les valeurs de tSIE ont été appréciées à partir d'un lot de 30 urines différentes. Dans le cas d'un tSIE inférieur à 250, il est préférable de décolorer l'échantillon par un passage sur une colonne de charbon actif.

Or, les tSIE obtenus dans les échantillons sont en majorité compris entre 286 et 450 , ce qui rend inutile, dans le cadre de notre méthode, le passage sur charbon actif.

\subsection{Activité et incertitudes}

Incertitude sur le rendement :

$$
\mathrm{Rdt}=\frac{\mathrm{Cps}-\mathrm{BF}}{A} \times \frac{1}{V} \times 100
$$

Cps : nombre de coups par seconde mesuré dans l'échantillon

$\mathrm{BF}$ : bruit de fond en coups par seconde

$A:$ activité théorique de l'échantillon en $\mathrm{Bq} \mathrm{ml}^{-1}$

$V$ : volume de prise d'essai en $\mathrm{ml}$.

D'après l'expression générale du rendement, l'erreur relative sur le rendement à $95 \%$ s'exprime de la façon suivante :

$$
\sigma_{\text {global }}=2 \sqrt{\sigma_{N}^{2}+\sigma_{\text {étalon }}^{2}+\sigma_{V}^{2}+\sigma_{\mathrm{BF}}^{2}}
$$

$\sigma_{\mathrm{BF}}$ : incertitude liée au bruit de fond (négligeable)

$\sigma_{N}$ : incertitude liée aux fluctuations de comptage $\left(\sigma_{N}=2 \sqrt{N} / N\right)$

$\sigma_{\text {ctalon }}$ : incertitude liée au titre de l'étalon $(0,83 \%)$

$\sigma_{v}$ : incertitude liée au volume de la prise d'essai (pipette précise à $0,4 \%$ ).

Ce qui donne une incertitude globale de $6 \%$. 
Incertitude sur l'activité :

$$
A=\frac{\mathrm{Cps}-\mathrm{BF}}{\mathrm{Rdt}} \times \frac{1}{V} \times 100
$$

Cps : nombre de coups par seconde mesuré dans l'échantillon

$\mathrm{BF}$ : bruit de fond en coups par seconde

$A$ : activité de l'échantillon en $\mathrm{Bq} \mathrm{ml}^{-1}$

$V:$ volume de prise d'essai en $\mathrm{ml}$

Rdt : rendement moyen.

D'après l'expression générale de l'activité, l'erreur relative sur l'activité à $95 \%$ s'exprime de la façon suivante :

$$
\begin{aligned}
& \sigma_{\text {global }}=2 \sqrt{\sigma_{\mathrm{sc}}+\sigma_{\mathrm{Rdt}}+\sigma_{V}} \\
& \text { avec: } \quad \sigma_{\mathrm{s}}=\sqrt{\sigma_{N}^{2}+\sigma_{\mathrm{BF}}^{2}}
\end{aligned}
$$

$\sigma_{\mathrm{S}}$ : incertitude qui prend en compte les incertitudes liées au bruit de fond et aux fluctuations de comptage

$\sigma_{\mathrm{Rdt}}$ : incertitude liée au rendement

$\sigma_{V}$ : incertitude liée au volume de la prise d'essai (pipette précise à $0,4 \%$ ).

L'incertitude globale sur l'activité est dépendante du niveau d'activité de l'échantillon : elle est de l'ordre de $6 \%$ pour une activité de $4 \mathrm{~Bq} \mathrm{ml}^{-1}$, de $10 \%$ pour une activité de $0,8 \mathrm{~Bq} \mathrm{ml}^{-1}$ et de $23 \%$ pour une activité de $0,08 \mathrm{~Bq} \mathrm{ml}^{-1}$.

\subsection{Limites de détection}

Les limites de détection sont évaluées à partir d'un bruit de fond moyen et un rendement moyen selon l'expression suivante (G.T. "Techniques analytiques », 1983) :

$$
\mathrm{LD}=4 \sqrt{\frac{2 \mathrm{BF}}{t}} \times \frac{100}{\mathrm{Rdt}}
$$

$\mathrm{LD}$ : limite de détection en $\mathrm{Bq}$

$\mathrm{BF}$ : bruit de fond en coups par seconde

$t$ : durée de la mesure en secondes

Rdt : rendement en \%.

Pour un comptage de $10 \mathrm{~min}$, la limite de détection est de l'ordre de $150 \times 10^{-3} \mathrm{~Bq} \mathrm{ml}^{-1}$. Pour un comptage d'une heure (temps habituel de mesure en scintillation liquide dans un laboratoire de radiotoxicologie), la limite de détection est évaluée à $60 \times 10^{-3} \mathrm{~Bq} \mathrm{ml}^{-1}$. 


\section{Discussion}

\section{1. Étude comparative des méthodes d'analyse de ${ }^{99} \mathrm{Tc}$}

Il est toujours difficile de concilier rapidité et limite de détection basse. En effet, la méthode par ICP-MS (Inductively Coupled Plasma Mass Spectrometry) est la méthode de choix pour obtenir des limites de détection performantes (LD entre 1,1 et $1,4 \times 10^{-3} \mathrm{~Bq} \mathrm{ml}^{-1}$ ) (Morita et al., 1991). Mais cette méthode reste pour l'instant coûteuse et lourde à mettre en place dans un laboratoire de surveillance radiotoxicologique de routine.

La scintillation liquide demeure la méthode la plus courante (Lawson et al., 1985). Certains auteurs, préconisent néanmoins, dans le cadre de cette technique, une extraction préalable du technétium (par co-précipitation au perchlorate de tetraphénylarsenium (Cattarin et al., 1985) ou par résine échangeuse d'ions sur colonne «Teva» d'Eichrom (Nevissi et al., 1994)). La prise d'essai pouvant être très supérieure à celle de notre méthode, la limite de détection se réduit d'autant pour une même durée de comptage : 15 à $30 \times 10^{-3} \mathrm{~Bq} \mathrm{ml}^{-1}$ au lieu de $60 \times 10^{-3} \mathrm{~Bq} \mathrm{ml}^{-1}$ pour notre méthode. En revanche toute extraction ou préconcentration occasionne un supplément de matériel, de réactifs, de temps et réduit d'autre part le rendement de la technique car celui-ci sera pondéré par un rendement chimique (en principe toujours inférieur à $100 \%$ ).

Notre méthode offre l'avantage d'un mode opératoire simple et rapide (quelques minutes). La durée de l'analyse n'est en fait conditionnée que par le temps de la mesure physique. Cet avantage n'affecte pas trop la valeur de la limite de détection, ce qui est appréciable dans le cas d'un accident de contamination où l'on sollicite souvent le laboratoire à fournir un résultat fiable dans les délais les plus brefs.

Ajoutons d'autre part, que la scintillation liquide est la méthode la moins coûteuse.

\subsection{Validité de la méthode en cas d'incorporation accidentelle unique}

La CIPR classe les oxydes, hydroxydes, halogénures et nitrates de technétium dans la classe d'inhalation $W$ et les autres composés chimiques du technétium dans la classe d'inhalation D (CIPR, 1979). La figure 4 présente le modèle biocinétique du technétium (CIPR, 1994). 


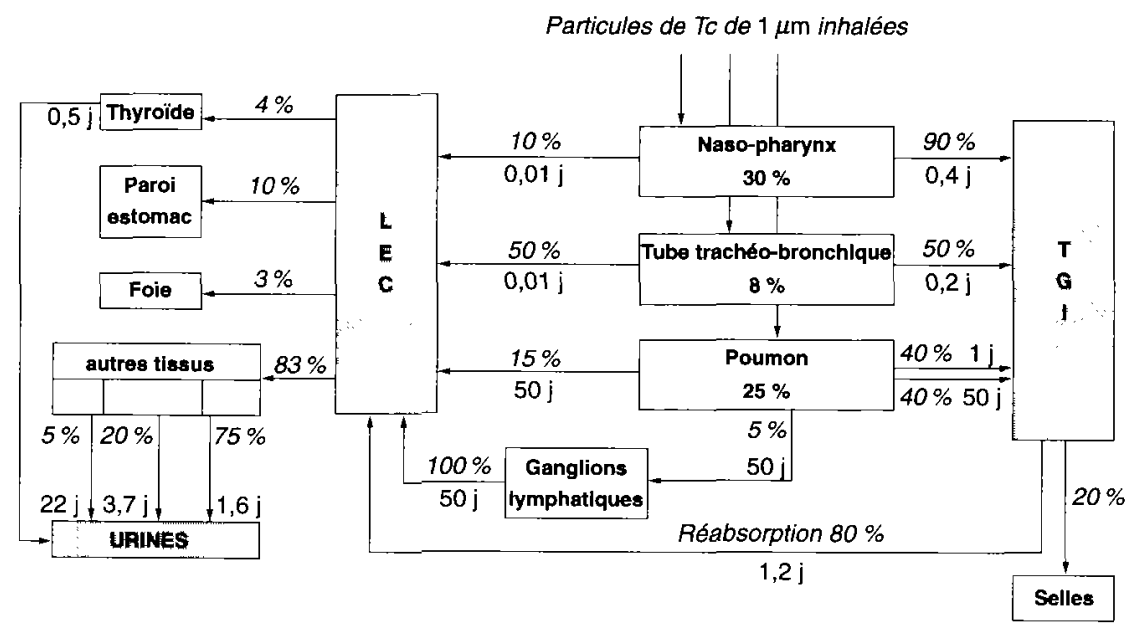

LEC : Liquide Extra-Cellulaire

TGI : Tube Gastro-Intestinal

$x \mathrm{j}$ : Période biologique d'élimination en jours.

Fig. 4. - Modèle biocinétique du technétium (composé de classe W).

Technecium biokinetic model (W class product).

Ce modèle va nous permettre d'évaluer approximativement le pourcentage de l'activité inhalée que l'on retrouve dans les urines au cours de deux analyses d'urines bien déterminées dans le temps:

- urines recueillies au cours des 48 premières heures après l'inhalation,

- urines de 24 heures à $t=183$ jours, c'est à dire lors de la première visite semestrielle de catégorie A depuis la contamination.

Soit une contamination de $100 \mathrm{~Bq}$ par inhalation :

Activité des urines de 48 heures après inhalation.

Sur le modèle biocinétique, on ne prend en compte, pour l'instant, que les transferts à courtes périodes biologiques. On considère d'autre part que ces périodes sont si faibles qu'à $t=0$, celles-ci peuvent être négligées. On suppose donc que toute la radioactivité inhalée atteint les différents organes instantanément. Cette hypothèse surestimera l'activité calculée dans les urines. Pour corriger ceci, nous ne tiendrons pas compte du compartiment «autres tissus» qui présente une période biologique de 3,7 jours. 
Les compartiments en amont des urines sont la thyroïde et le compartiment «autres tissus» $(75 \%)$. À $t=0$, ils reçoivent respectivement $1,6 \mathrm{~Bq}$ et $33 \mathrm{~Bq}$. Ils sont affectés d'une période d'élimination propre. De ce fait, à $t=48 \mathrm{~h}$, l'activité résiduelle dans ces deux compartiments est, respectivement, $0,1 \mathrm{~Bq}$ et $10,5 \mathrm{~Bq}$. Ainsi la variation totale d'activité pour ces deux compartiments au cours des 48 heures est de $17 \mathrm{~Bq}$. Cette activité se retrouve dans les urines recueillies au cours de ces 48 heures. Cette activité urinaire représente $10 \%$ de l'activité inhalée.

Activité des urines de 24 heures 6 mois après (surveillance radiotoxicologique catégorie A).

Sur les $25 \%$ de ${ }^{99} \mathrm{Tc}$ inhalé qui a atteint le poumon, $60 \%$ est redistribué, selon une période biologique de 50 jours, vers d'autres compartiments (poumon, ganglions lymphatiques, tractus gastro-intestinal et liquide extra-cellulaire). On considère qu'au bout de 6 mois, poumon, ganglions lymphatiques et tractus gastro-intestinal sont en équilibre : les éliminations dans un organe sont compensées par les apports. Et c'est en fait $15 \%(\mathrm{P})+5 \%(\mathrm{gg})+[40 \% \times 80 \%]$ (TGI) qui reviennent au LEC.

Ainsi, sur $100 \mathrm{~Bq}$ inhalés, $25 \mathrm{~Bq}$ ont atteint le poumon dont $15 \mathrm{~Bq}$ affectés d'une période biologique de 50 jours. À $t=183$ jours (6 mois), le poumon présentera une activité de $1,029 \mathrm{~Bq}$ et à $t=184$ jours $(6$ mois $+24 \mathrm{~h})$, une activité de $1,014 \mathrm{~Bq}$. Donc en 24 heures, s'élimine du poumon $1,5 \times 10^{-2} \mathrm{~Bq}$ qui se retrouve, via le LEC au niveau de la thyroïde, du foie, de la paroi de l'estomac et du compartiment «autres tissus». Seuls les compartiments thyroïde et «autres tissus», affectés de leur propre période biologique, excréteront, au niveau des urines de 24 heures, $1,3 \times 10^{-2} \mathrm{~Bq}$, soit $1,3 \times 10^{-2} \%$ de l'activité inhalée.

Ainsi, on peut admettre qu'au cours d'une contamination par inhalation, $10 \%$ de l'activité inhalée se retrouvent dans le prélèvement post-accidentel d'urine de 48 heures et $1,3 \times 10^{-2} \%$ dans l'échantillon d'urine de 24 heures, prélevé 6 mois après, au cours de la visite semestrielle de catégorie A.

Relier l'activité urinaire à l'activité inhalée est possible en considérant, à partir du modèle biocinétique décrit précédemment:

- que $10 \%$ de l'activité inhalée se retrouve dans les urines des 48 premières heures après l'incorporation,

- et que l'excrétion urinaire journalière de l'homme standard est de $1400 \mathrm{ml}$ (CIPR 23).

Ainsi une activité de $60 \times 10^{-3} \mathrm{~Bq} \mathrm{~m}^{-1}$ dans les urines des premières 48 heures (c'est-à-dire la valeur de notre limite de détection) représente une inhalation de $1680 \mathrm{~Bq}$ de technétium 99, soit $8,4 \times 10^{-5} \mathrm{LAI}$. 
Dans le cas d'un raisonnement inverse, le niveau d'enregistrement d'une expertise, fixé à $1 / 30$ de la LAI, correspond à environ $20 \mathrm{~Bq} \mathrm{ml}^{-1}$ dans les urines de 48 heures. Le niveau d'investigation, fixé à $1 / 10$ de la LAI correspond à $50 \mathrm{~Bq} \mathrm{ml}{ }^{-1}$ dans les urines de 48 heures. On voit que la limite de détection de la méthode est suffisamment basse pour appréhender ces niveaux de référence dérivés qui permettent de standardiser la conduite à tenir en fonction de l'importance présumée de la contamination.

\section{Conclusion}

La méthode proposée requiert peu de manipulations et aucune préconcentration, ce qui lui confère simplicité, rapidité et coût réduit. En outre, pour une technique par scintillation liquide, la mesure du technétium présente l'avantage d'être peu dépendante des phénomènes de quenching.

Enfin, la méthode est suffisamment sensible pour apprécier $8,4 \times 10^{-5} \mathrm{LAI}$ ainsi que les niveaux d'enregistrement et d'investigation indispensables à une surveillance standardisée. Cette méthode est d'ailleurs actuellement réalisée en routine au Laboratoire d'Analyses de Biologie Médicale du Centre d'Études de Cadarache dans le cadre d'une surveillance systématique à certains postes de travail.

\section{RÉFÉRENCES}

Cattarin S., Doretti L., Mazzi U. (1985) Determination of ${ }^{99} \mathrm{Tc}$ in urine by liquid scintillation counting to evaluate internal contamination, Health Phys. 49, 795.

CIPR (1979) Annals of the ICRP : Limits for intakes of radionuclides by workers. ICRP publication 30, part 1. Pergamon Press: Oxford.

CIPR (1979) Annals of the ICRP : Statement and recommendations of the 1980 Brighton Meeting of the ICRP. ICRP publication 30, part 2. Pergamon Press : Oxford.

CIPR (1994) Annals of the ICRP : Human respiratory tract model for radiological protection. ICRP publication 66. Elsevier: Oxford.

GROUPE DE TRAVAIL «TECHNIQUES ANALYTIQUES» (1983) Limite de détection d'un signal dans un bruit de fond, application aux mesures de radioactivité par comptage. Rapport CEA-R5201.

GMELIN (1982) Handbook of inorganic chemistry : Tc, supplement vol. 1. Springer Verlag 8th ed. : Berlin, Heidelberg, New York, p. 78, pp. 214-217.

Lawson B.L., Powell C.R., Pinkerton T.C. (1985) Quantification of ${ }^{99} \mathrm{Tc}$ by liquid scintillation counting : an evaluation of chemical quenching by liquid chromatographic eluents, J. Radioanal. Nucl. Chem. Lett. 94, 71.

Morita S., Kim C.K., Takaku Y., Seki R., Ikeda N. (1991) Determination of technecium 99 in environmental samples by inductively coupled plasma mass spectrometry. Appl. Radiat. Isot. 42 (6), 531-534.

Nevissi A.E., Silverston M., Strebin R.S., Kaye J.H. (1994) Radiochemical determination of technecium 99. J. Radioanal. Nucl. Chem. 177 (1), 91-99.

Simonet G., Oria M. (1980) Les mesures de radioactivité à l'aide de compteurs à scintillateur liquide. Eyrolles Editions: Paris, pp. 108-128. 